\title{
Dynamo generated field emergence through recurrent plasmoid ejections
}

\author{
Jörn Warnecke ${ }^{1,2}$ and Axel Brandenburg ${ }^{1,2}$ \\ ${ }^{1}$ Nordita, AlbaNova University Center, \\ Roslagstullsbacken 23, SE-10691 Stockholm, Sweden \\ email: joern@nordita.org \\ ${ }^{2}$ Department of Astronomy, AlbaNova University Center, \\ Stockholm University, SE 10691 Stockholm, Sweden
}

\begin{abstract}
Magnetic buoyancy is believed to drive the transport of magnetic flux tubes from the convection zone to the surface of the Sun. The magnetic fields form twisted loop-like structures in the solar atmosphere. In this paper we use helical forcing to produce a large-scale dynamogenerated magnetic field, which rises even without magnetic buoyancy. A two layer system is used as computational domain where the upper part represents the solar atmosphere. Here, the evolution of the magnetic field is solved with the stress-and-relax method. Below this region a magnetic field is produced by a helical forcing function in the momentum equation, which leads to dynamo action. We find twisted magnetic fields emerging frequently to the outer layer, forming arch-like structures. In addition, recurrent plasmoid ejections can be found by looking at space-time diagrams of the magnetic field. Recent simulations in spherical coordinates show similar results.
\end{abstract}

Keywords. MHD, Sun: magnetic fields, Sun: coronal mass ejections (CMEs), turbulence

\section{Introduction}

The solar magnetic field is broadly believed to be in the form of concentrated flux ropes in the bulk of the convection zone. At the solar surface they emerge to form bipolar regions and sunspots in the photosphere that appear as twisted loop-like structures in the higher atmosphere. However, there is no clear evidence that magnetic fields are generated in flux tubes that emerge from the tachocline all the way to the surface of the Sun. Numerical simulations have successfully shown that magnetic buoyancy, which has been thought to be the main driver of flux tube emergence, can be efficiently suppressed by downward pumping due to the stratification with concentrated downdraft in the solar convection zone (Nordlund et al. 1992; Tobias et al. 1998). Large-scale dynamo simulations suggest that flux tubes are primarily a feature of the kinematic dynamo regime, but tend to be less pronounced in the nonlinear stage (Käpylä et al. 2008). An alternative mechanism might simply be the relaxation of strongly twisted magnetic fields reaching the surface of the Sun. Twisted magnetic fields are produced by a large-scale dynamo mechanism which is generally believed to be the source of solar magnetic activity (Parker 1979). In order to study the emergence of helical magnetic fields from a dynamo, we consider a model that combines a direct simulation of a turbulent large-scale dynamo with a simple treatment for the evolution of nearly force-free magnetic fields above the surface of the dynamo. In the context of force-free magnetic field extrapolations this method is also known as the stress-and-relax method (Valori et al. 2005). Including a nearly forcefree field in the upper part of the domain has the additional benefit of allowing a more realistic modeling of the dynamo itself. This is important, because it is known that the properties of the large-scale magnetic field depend strongly on the boundary conditions. 
In the upper atmosphere, direct numerical simulation of the solar corona show force-free magnetic fields (Gudiksen \& Nordlund 2005).

Above the solar surface, we expect helical magnetic fields to drive flares and coronal mass ejections through the Lorentz force. In the present paper we highlight some of the main results of our earlier work (Warnecke \& Brandenburg 2010) and present recent applications and results using spherical coordinates.

\section{The Model}

A two layer system is used, where the upper part is modelled as a nearly force-free magnetic field by using the stress-and-relax method (Valori et al. 2005) and in the lower part a dynamo field is generated through helically forced turbulence. We combine these two layers by simply turning off terms that should not be included in the upper part of the domain. We do this with error function profiles of the form

$$
\theta_{w}(z)=\frac{1}{2}\left(1-\operatorname{erf} \frac{z}{w}\right)
$$

where $w$ is the width of the transition.

\subsection{Stress-and-relax method}

The equation for the velocity in the stress-and-relax method is similar to the usual momentum equation, except that there is neither pressure, nor gravity, nor other driving forces on the right-hand side, so we just have

$$
\frac{\mathrm{d} \boldsymbol{U}}{\mathrm{d} t}=\boldsymbol{J} \times \boldsymbol{B} / \rho+\boldsymbol{F}_{\text {visc }}
$$

where $\boldsymbol{J} \times \boldsymbol{B}$ is the Lorentz force, $\boldsymbol{J}=\boldsymbol{\nabla} \times \boldsymbol{B} / \mu_{0}$ is the current density, $\mu_{0}$ is the vacuum permeability, $\boldsymbol{F}_{\text {visc }}$ is the viscous force, and $\rho$ is here treated as a constant that determines the strength of the velocity correction. Equation (2.2) is solved together with the uncurled induction equation,

$$
\frac{\partial \boldsymbol{A}}{\partial t}=\boldsymbol{U} \times \boldsymbol{B}+\eta \nabla^{2} \boldsymbol{A}
$$

with $\eta$ being the magnetic diffusivity.

\subsection{Forced dynamo region}

In the lower part the velocity is driven by a forcing function and the density is evolved using the continuity equation,

$$
\frac{\mathrm{d} \boldsymbol{U}}{\mathrm{d} t}=-\nabla h+\boldsymbol{f}+\boldsymbol{J} \times \boldsymbol{B} / \rho+\boldsymbol{F}_{\mathrm{visc}}, \quad \frac{\mathrm{d} h}{\mathrm{~d} t}=-c_{s}^{2} \boldsymbol{\nabla} \cdot \boldsymbol{U},
$$

where $\boldsymbol{F}_{\text {visc }}$ is the viscous force, $h=c_{\mathrm{S}}^{2} \ln \rho$ is the specific pseudo-enthalpy, $c_{\mathrm{S}}=$ const is the isothermal sound speed, and $f$ is a forcing function that drives turbulence in the interior and consists of random plane helical transverse waves with an average forcing wavenumber $k_{\mathrm{f}}$. The pseudo-enthalpy $h$ is given by $\rho^{-1} \nabla p=c_{\mathrm{S}}^{2} \boldsymbol{\nabla} \ln \rho=\nabla h$. Equations (2.4) are solved together with the induction Eqs. (2.3).

The simulation box is horizontally periodic. For the magnetic field we adopt verticalfield and perfect-conductor conditions at the top and bottom boundaries, respectively. For the velocity we employ stress-free conditions at both boundaries. In this paper we present direct numerical simulations using the PENCIL CoDE†, a modular high-order 


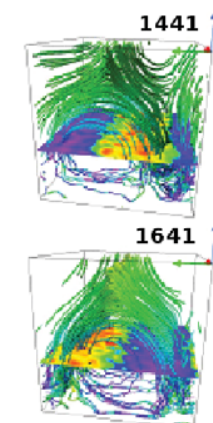

$\tau=2761$

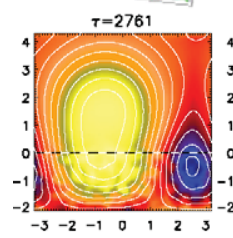

$\tau=2921$

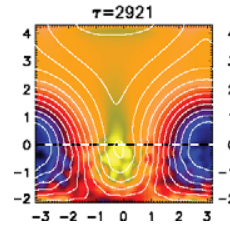

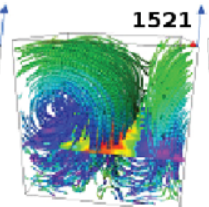

1721

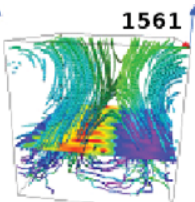

1801

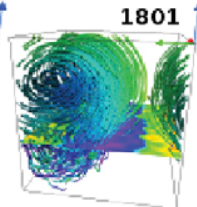

$T=2841$

$\tau=2801$

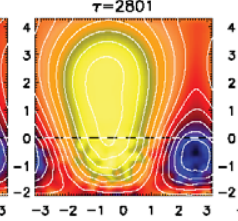

$\tau=2961$

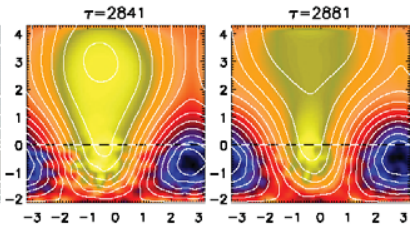

$\tau=3001$

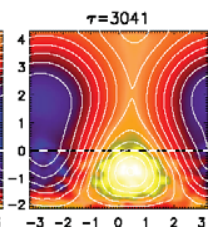

Figure 1. Upper part: Time series of arcade formation and decay. Field lines are colored by their local field strength which increases from purple to green. The inclined plane in the box shows $B_{z}$ increasing from red (positive) to purple (negative). The normalized time $\tau$ is giving in each panel. Lower part: Time series of the formation of a plasmoid ejection. Contours of $\left\langle A_{x}\right\rangle_{x}$ are shown together with a color-scale representation of $\left\langle B_{x}\right\rangle_{x}$; dark/blue stands for negative and light/yellow for positive values. The contours of $\left\langle A_{x}\right\rangle_{x}$ correspond to field lines of $\langle\boldsymbol{B}\rangle_{x}$ in the $y z$ plane. The dotted horizontal lines show the location of the surface at $z=0$. Adapted from Warnecke \& Brandenburg (2010).

code (sixth order in space and third-order in time) for solving a large range of partial differential equations.

\section{Results}

After a period of exponential growth, the magnetic field saturates at $78 \%$ of the equipartition field strength, $B_{\text {eq }}$ in the turbulent layer. This behavior is typical for forced dynamo action. The structure of the magnetic field is a large-scale field in the turbulent zone. It always shows a systematic variation in one of the two horizontal directions. It is a matter of chance whether this variation is in the $x$ or in the $y$ direction. After the saturation phase the magnetic field extends well into the upper layer where it tends to produce an arcade-like structure, as seen in the upper panel of Fig. 1. The arcade opens up in the middle above the line where the vertical field component vanishes at the surface. This leads to the formation of anti-aligned field lines with a current sheet in the middle. The dynamical evolution is clearly seem in a sequence of field line images in the upper panel of Fig. 1, where anti-aligned vertical field lines reconnect above the neutral line and form a closed arch with plasmoid ejection. This arch then changes its connectivity at the foot points in one of the two horizontal directions (here the $y$ direction), making the field lines bulge upward to produce a new reconnection site with anti-aligned field lines some distance above the surface. Field line reconnection is best seen for two-dimensional 

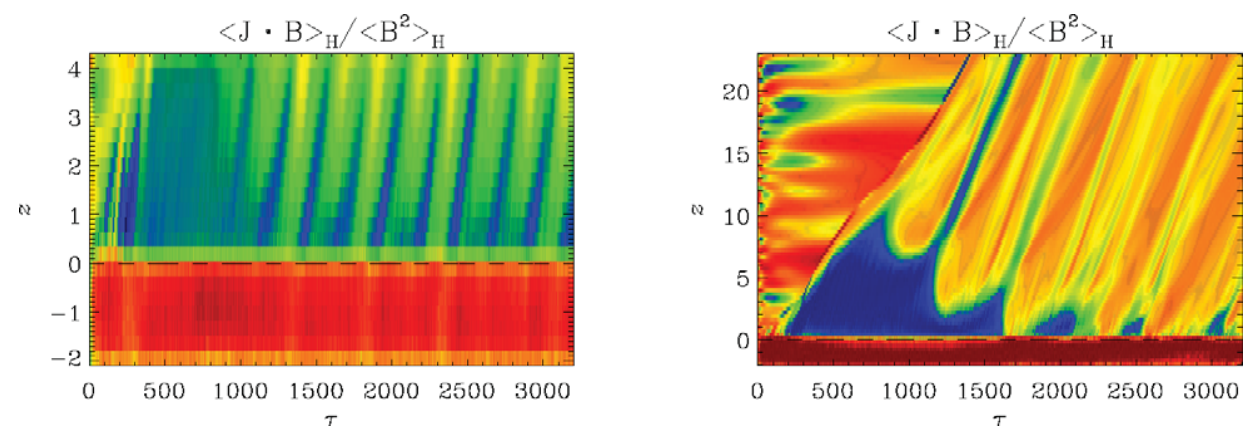

Figure 2. Left panel: Dependence of $\langle\boldsymbol{J} \cdot \boldsymbol{B}\rangle_{\mathrm{H}} /\left\langle\boldsymbol{B}^{2}\right\rangle_{\mathrm{H}}$ versus time $\tau$ and height $z$ for $L_{z}=6.4$ with $\operatorname{Re}_{M}=3.4$. Right panel: Similar to the left panel, but for $L_{z}=8 \pi$ and $\operatorname{Re}_{M}=6.7$. Adapted from Warnecke \& Brandenburg (2010).
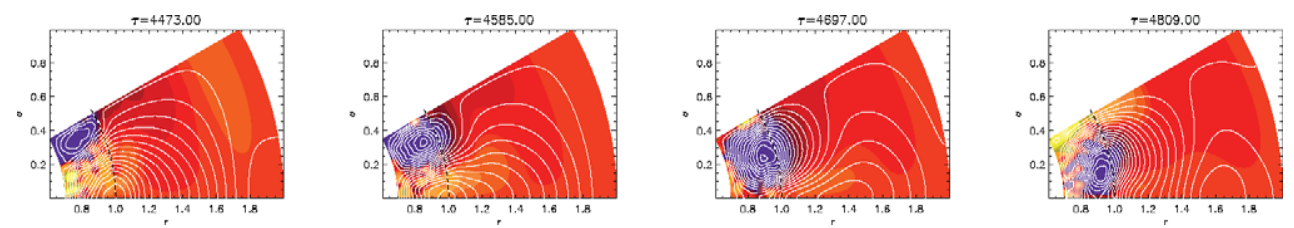

Figure 3. Time series of flux emergence in spherical coordinates. Contours of $r \sin \theta\left\langle A_{\phi}\right\rangle_{\phi}$ are shown together with a color-scale representation of $\left\langle B_{\phi}\right\rangle_{\phi}$; dark/blue stands for negative and light/yellow for positive values. The contours of $r \sin \theta\left\langle A_{\phi}\right\rangle_{\phi}$ correspond to field lines of $\langle\boldsymbol{B}\rangle_{\phi}$ in the $r \theta$ plane. The dotted horizontal lines show the location of the surface at $r=1$ solar radii.

magnetic fields, because it is then possible to compute a flux function whose contours correspond to field lines in the corresponding plane. In the present case the large-scale component of the magnetic field varies little in the $x$ direction, so it makes sense to visualize the field averaged over $x$ (see lower panel of Fig. 1).

In order to demonstrate that plasmoid ejection is a recurrent phenomenon, it is convenient to look at the evolution of the ratio $\langle\boldsymbol{J} \cdot \boldsymbol{B}\rangle_{\mathrm{H}} /\left\langle\boldsymbol{B}^{2}\right\rangle_{\mathrm{H}}$ versus $t$ and $z$. This is done in right panel of Fig. 2 for $L_{z}=6.4$ and $\operatorname{Re}_{M}=3.4$ and in the left panel of Fig. 2 for $L_{z}=8 \pi$ and $\operatorname{Re}_{M}=6.7$. It turns out that in both cases the typical speed of plasmoid ejecta is about half the rms velocity of the turbulence in the interior region.

As an example of further work in this direction, we also present in this paper magnetic flux emergence in spherical coordinates. The dynamical evolution can be seen in Fig. 3, where a modulated slice covers the convection zone from 0.7 solar radii through the upper atmosphere to two solar radii. This meridional slice of a sphere consists, like the simulation box above, of two layers which contain the same physical properties. We solve the same equations as described in Section 2. Again, there is flux emerge through the surface above the turbulence zone. This can be seen as a recurrent event. Unfortunately, reconnection, current sheets and plasmoid ejections have not been seen in the present setup, although there are indications that they do occur in even more recent spherical models where gravity and density stratification are included (Warnecke et al. 2011).

Our first results are promising in that the dynamics of the magnetic field in the exterior is indeed found to mimic open boundary conditions at the interface between the turbulence zone and the exterior at $z=0$. In particular, it turns out that a twisted magnetic field generated by the helical dynamo beneath the surface is able to produce flux emergence in ways that are reminiscent of that found in the Sun. The first results in spherical coordinates show recurrent flux emergence, but plasmoid ejections in a curved environment may only be possible if gravity and density stratification are included. 


\section{References}

Gudiksen, B. V. \& Nordlund. 2005, Astrophys. J., 618, 1031

Käpylä, P. J., Korpi, M. J., \& Brandenburg, A. 2008, Astron. Astrophys, 491, 353

Nordlund, Å., Brandenburg, A., Jennings, R. L., Rieutord, M., Ruokolainen, J., Stein, R. F., \& Tuominen, I. 1992, Astrophys. J., 392, 647

Parker, E. N. 1979, Cosmical magnetic fields (Clarendon Press, Oxford)

Tobias, S. M., Brummell, N. H., Clune, T. L., \& Toomre, J.1998, Astrophys. J. Lett., 502, L177

Valori, G., Kliem, B., \& Keppens, R. 2005, Astron. Astrophys, 433, 335

Warnecke, J. \& Brandenburg, A. Astron. Astrophys, 2010, 523, A19

Warnecke, J., Brandenburg, A., \& Mitra, D., 2011, A\&A (submitted), arXiv:1104.0664 